\title{
The Spectral Gap of a Random Subgraph of a Graph
}

\author{
Fan Chung and Paul Horn
}

Abstract. We examine the relationship of a graph $G$ and its random subgraphs, which are defined by independently choosing each edge with probability $p$. Suppose that $G$ has a spectral gap $\lambda$ (in terms of its normalized Laplacian) and minimum degree $d_{\min }$. Then we can show that a random subgraph of $G$ on $n$ vertices with edge-selection probability $p$ almost surely has as its spectral gap $\lambda-O\left(\sqrt{\frac{\log n}{p d_{\min }}}+\frac{(\log n)^{3 / 2}}{p d_{\min }(\log \log n)^{3 / 2}}\right)$.

\section{Introduction}

Often, when we examine a large graph, perhaps arising from some realistic setting (e.g., webgraphs, biological networks, or some information network derived from a large database), we are unable to see the entire graph. Instead, what we can observe are relatively small subgraphs of the large graph. We are interested, then, in understanding the relationship between the large host graph and the subgraphs that we actually observe. The basic question becomes whether there are some graph invariants that we can evaluate for the original host graph that lead to good estimates for properties and structures of our observed subgraphs, and vice-versa.

In this paper, we begin by considering a host graph $G$. Fixing an edge-selection parameter, $p \in[0,1]$, we consider the family of subgraphs by percolating $G$ with parameter $p$. That is, our observed subgraph $H$ is a random subgraph of $G$ such that each edge from $G$ is chosen independently with the edge-selection probability $p$.

(C) A K Peters, Ltd. I542-795I/07 \$0.50 per page 
An example of the utility of such an approach is in the study of epidemiological models. Here, the host graph represents a contact network, where the vertices represent people in some community of interest, and edges denote certain contact or interaction among pairs of people. A disease is often considered to pass through an interaction with probability $p$. Thus, the observed random subgraph can represent the actual spread of disease through the contact network. Other examples concern various social networks, such as telephone or instant messaging networks. A group of friends contacting each other during a specified period of time in a large social network can be viewed as a random subgraph of a large host graph consisting of all contacts between members of the network. The classical Erdős-Rényi model, $G(n, p)$, is also a particular instance of this model. Indeed, it is the special case where the host graph is the complete graph $K_{n}$.

In this paper, we consider the relationship of the spectrum of the host graph and that of a random subgraph with edge-selection probability $p$. The methods that we use here are based on Wigner's high moment methods [Wigner 58]. Such an approach was extensively utilized in the early work on random graphs and matrices in numerous research papers including the early work by Füredi and Komlós [Füredi and Komlós 81] as well as in some recent work on random sparse graphs in [Chung et al. 04] and [Vu 07]. These previous works belong, however, to the special case in which the host graph is taken to be the complete graph (or the full matrix). Here, similar techniques are used and modified in order to deal with the spectral gap of a random subgraph of a given host graph. Nachmias and Peres [Nachmias and Peres 08] have studied properties related to the spectrum, in particular diameter and mixing time, in percolated regular graphs. Similarly, Ofek [Ofek 07] has studied expansion in the giant component of percolated pseudorandom graphs. Several other authors have studied several properties of percolated finite graphs. In particular, Alon et al. [Alon et al. 04] and Frieze et al. [Frize et al. 04] have studied the emergence of the giant component in expanders, with Alon et al. also studying isoperimetric properties.

Since we are dealing with a general graph (with possibly uneven degree distribution), we consider the (normalized) Laplacian (see [Chung 97]). For a graph on $n$ vertices, the Laplacian is a symmetric matrix of size $n \times n$ defined as follows (more details will be given in the next section):

$$
\mathcal{L}=I-D^{-1 / 2} A D^{-1 / 2}
$$

where $A$ denotes the adjacency matrix and $D$ denotes the diagonal degree matrix. Let

$$
0=\lambda_{0} \leq \lambda_{1} \leq \ldots \leq \lambda_{n-1}
$$


denote the eigenvalues of $\mathcal{L}$. We write

$$
\lambda=\min \left\{\lambda_{1}, 2-\lambda_{n-1}\right\} .
$$

For example, a random $d$-regular graph on $n$ vertices almost surely has a spectral gap $\lambda>1-(2 \sqrt{d-1}+\epsilon) / d$ for any positive constant $\epsilon$ as $n$ approaches infinity (see [Friedman 03]).

When there is a nontrivial spectral gap, i.e., when $\lambda$ is separated from 0 , the graph has many nice properties, such as expansion properties and the rapid convergence of random walks on the graph. What we will show here is how to derive a bound for the spectral gap of a random subgraph of $G$ in terms of the probability $p$ of edge-selection and the spectral gap of $G$.

Theorem I.I. Suppose $G$ is a graph on $n$ vertices with spectral gap $\lambda$ and minimum degree $d_{\text {min. }}$ A random subgraph $H$ of $G$ with edge-selection probability $p$ almost surely has a spectral gap $\lambda_{H}$ satisfying

$$
\lambda_{H}=\lambda-O\left(\sqrt{\frac{\log n}{p d_{\min }}}+\frac{(\log n)^{3 / 2}}{p d_{\min }(\log \log n)^{3 / 2}}\right) .
$$

An equivalent statement for the above theorem is the following: For $p d_{\min } \geq$ $(\log n)^{2} /(\log \log n)^{3}$, we have

$$
\lambda_{H}=\lambda-O\left(\sqrt{\frac{\log n}{p d_{\min }}}\right),
$$

and, for $p d_{\min }<(\log n)^{2} /(\log \log n)^{3}$, we have

$$
\lambda_{H}=\lambda-O\left(\frac{(\log n)^{3 / 2}}{p d_{\min }(\log \log n)^{3 / 2}}\right) .
$$

As an immediate consequences of the above theorem, a random graph almost surely will have the following properties (see [Chung 97, Krivelevich and Sudakov 06]):

Corollary 1.2. For a graph $G$ on $n$ vertices with spectral gap $\lambda$ and minimum degree $d_{\text {min }}$, a subgraph $H$ with edge-selection probability $p$ almost surely satisfies the following properties:

1. $H$ satisfies the expansion property as follows: For $X \subseteq V(H)$, the number of edges in $H$ leaving $X$, denoted by $\partial_{H}(X)$ satisfies

$$
\partial_{H}(X) \geq\left(\lambda-O\left(\sqrt{\frac{\log n}{p d_{\min }}}+\frac{(\log n)^{3 / 2}}{p d_{\min }(\log \log n)^{3 / 2}}\right)\right) p \operatorname{vol}(X),
$$


where $\operatorname{vol}(X)$ is $\sum_{x \in X} d(x)$ and $d(x)$ denotes the degree of $x$ in the host graph $G$.

2. $H$ satisfies the discrepancy property as follows: For $X, Y \subseteq V(H)$, the number of edges of $H$ between $X$ and $Y$, denoted by $e_{H}(X, Y)$, satisfies

$$
\begin{aligned}
& \left|e_{H}(X, Y)-p \frac{\operatorname{vol}(X) \operatorname{vol}(Y)}{\operatorname{vol}(H)}\right| \\
& \quad \leq\left(1-\lambda-O\left(\sqrt{\frac{\log n}{p d_{\min }}}+\frac{(\log n)^{3 / 2}}{p d_{\min }(\log \log n)^{3 / 2}}\right)\right) p \sqrt{\operatorname{vol}(X) \operatorname{vol}(Y)} .
\end{aligned}
$$

3. For a random walk on $H$ with transition probability matrix $P_{H}$, the total variation distance after $t$ steps from the stationary distribution $\pi$, denoted by $\Delta_{T V}(t)$, is bounded above by

$$
\begin{aligned}
\Delta_{T V}(t) & =\max _{A \subseteq V} \max _{y \in V}\left|\sum_{x \in A}\left(P_{H}^{t}(y, x)-\pi(x)\right)\right| \\
& \leq e^{-c}
\end{aligned}
$$

for any $c>0$, provided $t$ satisfies

$$
t \geq \frac{1}{\lambda-O\left(\sqrt{\frac{\log n}{p d_{\min }}}+\frac{(\log n)^{3 / 2}}{p d_{\min }(\log \log n)^{3 / 2}}\right)}\left(\log \frac{\operatorname{vol}(H)}{\min _{x} d(x)}+c\right) .
$$

The above theorem depends on the volume $\operatorname{vol}(X)$ of a subset $X$ in $G$. It can also be thought of, however, as depending on the volume of $X$ in $H$ in the following manner. The volume of $X$ in $H$ is denoted by $\operatorname{vol}_{H}(X)=\sum_{x \in X} d_{H}(x)$, where $d_{H}(x)$ is the degree of $x$ in the subgraph $H$. If the volume of $X$ in $G$ is large, we have good control over the volume of $X$ in $H$ using Chernoff bounds. It is not difficult to prove that if $\operatorname{Vol}(X)$ is sufficiently large, we almost surely have

$$
\left|\operatorname{vol}_{H}(X)-p \operatorname{vol}(X)\right| \leq g(n) \sqrt{p \operatorname{vol}(X)}
$$

for any function $g(n)$ that goes to infinity with $n$ (see [Chung and Lu 06]).

\section{Preliminaries}

Let $G=(V, E)$ be a graph. We denote by $H$ a random graph obtained from $G$ by taking each edge independently with probability $p$. That is,

$$
\operatorname{Pr}(\{u, v\} \in E(H))= \begin{cases}p & \text { if }\{u, v\} \in E(G), \\ 0 & \text { otherwise. }\end{cases}
$$


Let $A$ and $A_{H}$ denote the adjacency matrix of $G$ and $H$, respectively. We denote the diagonal matrices whose entries consist of the degrees of the vertices in $G$ and $H$, respectively, by $D$ and $D_{H}$. Let $0=\eta_{0} \leq \cdots \leq \eta_{n-1}$ denote the eigenvalues of $\mathcal{L}_{H}$, and let $\varphi_{i}$ for $i=0, \ldots, n-1$ denote a set of orthonormal eigenvectors associated with the $\eta_{i}$ (represented here as row vectors). The projection to $\varphi_{i}$, for each $i$, is $P_{i}=\varphi_{i}^{*} \varphi_{i}$ where $\varphi^{*}$ denotes the transpose of $\varphi$. Then we have

$$
\mathcal{L}_{H}=\sum_{i} \eta_{i} P_{i}
$$

We consider, then, the matrix

$$
\begin{aligned}
M & =I-\mathcal{L}_{H}-P_{0} \\
& =\sum_{i \neq 0}\left(1-\eta_{i}\right) P_{i} .
\end{aligned}
$$

We will use the fact that, for any integer $k$, we have

$$
\operatorname{Trace}\left(M^{2 k}\right)=\sum_{i \neq 0}\left(1-\eta_{i}\right)^{2 k} .
$$

Immediately, we have the following:

Fact 2.I. For any positive integer $k$

$$
\max _{i \neq 0}\left|1-\eta_{i}\right|=|| M \| \leq\left(\operatorname{Trace}\left(M^{2 k}\right)\right)^{1 /(2 k)} .
$$

We denote the spectral gap of $H$ by $\lambda_{H}=\max \left\{\eta_{1}, 2-\eta_{n-1}\right\}$. Hence we have

$$
\lambda_{H}=1-\max _{i \neq 0}\left|1-\eta_{i}\right|=1-\|M\| .
$$

Let $K$ denote the all ones matrix. We can rewrite $M$ as

$$
\begin{aligned}
M & =D_{H}^{-1 / 2} A_{H} D_{H}^{-1 / 2}-P_{0} \\
& =D_{H}^{-1 / 2} A_{H} D_{H}^{-1 / 2}-\varphi_{0}^{*} \varphi_{0} \\
& =D_{H}^{-1 / 2} A_{H} D_{H}^{-1 / 2}-\frac{1}{\operatorname{vol}(H)} D_{H}^{1 / 2} K D_{H}^{1 / 2} .
\end{aligned}
$$

Instead of directly dealing with $M$, we consider the simpler matrix

$$
\begin{aligned}
C & =p^{-1} D^{-1 / 2} A_{H} D^{-1 / 2}-\frac{1}{p \operatorname{vol}(G)} p D^{1 / 2} K D^{1 / 2} \\
& =p^{-1} D^{-1 / 2} A_{H} D^{-1 / 2}-\frac{1}{\operatorname{vol}(G)} D^{1 / 2} K D^{1 / 2}
\end{aligned}
$$


where one may note that $p \operatorname{vol}(G)$ is the expected volume of $H$.

In a way, $C$ can be thought of as an estimate for the expectation of $M$. Our plan is first to consider $\|C\|$ in the next section and then bound the norm of the difference between $M$ and $C$ in Section 4 .

\section{A Bound on $\|C\|$}

In this section we prove the following theorem.

Theorem 3.I. Let $G$ be a given graph with spectral gap $\lambda$ and minimum degree $d_{\min }$. Let $H$ be a random subgraph of $G$ with edge-selection probability $p$. Then the matrix $C$ as defined in (2.1) almost surely satisfies

$$
\|C\|=1-\lambda+O\left(\sqrt{\frac{\log n}{p d_{\min }}}+\frac{(\log n)^{3 / 2}}{p d_{\min }(\log \log n)^{3 / 2}}\right) .
$$

Proof. To bound the norm of $C$, we express $C$ as a sum of two parts:

$$
C=B+M^{\prime}
$$

where

$$
B=p^{-1} D^{-1 / 2} A_{H} D^{-1 / 2}-D^{-1 / 2} A D^{-1 / 2}
$$

and

$$
M^{\prime}=D^{-1 / 2} A D^{-1 / 2}-\frac{1}{\operatorname{vol}(G)} D^{1 / 2} K D^{1 / 2}
$$

Note that $M^{\prime}$ is equivalent to the matrix $M$ for the graph $G$. Hence we have

$$
\left\|M^{\prime}\right\|=1-\lambda \text {. }
$$

It suffices to show that almost surely we have

$$
\|B\|=O\left(\sqrt{\frac{\log n}{p d_{\min }}}+\frac{(\log n)^{3 / 2}}{p d_{\min }(\log \log n)^{3 / 2}}\right) .
$$

In other words, we wish to prove that, for any $\epsilon>0$, there is an absolute constant $c$ so that for $n$ sufficiently large, we can bound the probability as follows:

$$
\operatorname{Pr}\left[\|B\| \geq c\left(\sqrt{\frac{\log n}{p d_{\min }}}+\frac{(\log n)^{3 / 2}}{p d_{\min }(\log \log n)^{3 / 2}}\right)\right] \leq \epsilon .
$$


The matrix $B$ is a random matrix, where the entries $b_{i j}$ are independent random variables defined by

$$
b_{i j}= \begin{cases}\frac{1}{p \sqrt{d_{i} d_{j}}}-\frac{1}{\sqrt{d_{i} d_{j}}}=\frac{1-p}{p \sqrt{d_{i} d_{j}}} & \text { if } v_{i} \sim v_{j} \in H, \\ -\frac{p}{p \sqrt{d_{i} d_{j}}} & \text { if } v_{i} \nsim v_{j} \in H \text { and } v_{i} \sim v_{j} \in G, \\ 0 & \text { otherwise. }\end{cases}
$$

Here $d_{i}$ denotes the degree of $v_{i}$ in $G$. It follows from the definition that the expected value of $b_{i j}$ satisfies

$$
\mathrm{E}\left[b_{i j}\right]=0 \text {. }
$$

Now consider the $(i, i)$ th entry of $B^{2 k}$. A typical term of such an entry is of the form

$$
b_{i_{1} i_{2}} b_{i_{2} i_{3}} \ldots b_{i_{2 k-1} i_{2 k}}
$$

with $i_{1}=i_{2 k}=i$. Assuming this term is non-zero, this corresponds to a closed walk in $G$ starting and ending at $v_{i}$. Taking expectations, we note that $E\left[b_{i_{1} i_{2}} b_{i_{2} i_{3}} \ldots b_{i_{2 k-1} i_{2 k}}\right] \neq 0$ only if each $b_{i j}$ occurs at least twice (since $\mathrm{E}\left[b_{i j}\right]=0$ and all $b_{i j}$ 's are independent). In other words, each edge must occur at least twice in the closed walk. We refer to a such a closed walk, which contributes to the expected trace, as a surviving walk.

To determine the expected contribution of a surviving walk to $\operatorname{Trace}\left(B^{2 k}\right)$, we consider the expected value $\mathrm{E}\left[b_{i j}^{m}\right]$. Note that, for $m \geq 2$,

$$
\left|\mathrm{E}\left[b_{i j}^{m}\right]\right| \leq \frac{\left|(1-p)^{m} p+(-p)^{m}(1-p)\right|}{p^{m}\left(d_{i} d_{j}\right)^{m / 2}} \leq \frac{p}{p^{m}\left(d_{i} d_{j}\right)^{m / 2}}=\frac{1}{p^{m-1}\left(d_{i} d_{j}\right)^{m / 2}} .
$$

The last inequality follows from the easy fact that $\left|(1-p)^{m} p+(-p)^{m}(1-p)\right| \leq p$ when $p \leq 1$.

To bound the trace of $B^{2 k}$, we must get a handle on the number of surviving walks and their contribution to the trace. Consider a surviving walk of length $2 k$ on vertices $v_{1}, \ldots, v_{l+1}$, and let us assume that the vertices are labelled by their first occurrence in the walk. Thus, to get to vertex $v_{i}$, we must have followed an edge from one of $v_{1}, \ldots, v_{i-1}$. We define the exposure sequence of the walk to be a vector $\left(a_{1}, \ldots, a_{l}\right)$ such that we first travel to vertex $v_{i}$ from vertex $v_{a_{i-1}}$. Clearly, $a_{i} \in\{1, \ldots, i\}$. Hence there are at most $l$ ! possible exposure sequences. We seek to enumerate our surviving walks by their exposure sequences.

Consider a surviving walk on vertices $v_{1}, \ldots, v_{l+1}$ with exposure sequence $\left(a_{1}, \ldots, a_{l}\right)$. Let us assume that the walk contains edges $e_{1}, \ldots, e_{k}$ with multiplicities $m_{1}, \ldots, m_{k}$, respectively. Then the contribution of the walk to the 
expected value of the trace is at most

$$
\begin{aligned}
\mathrm{E}\left[b_{e_{1}}^{m_{1}} \ldots b_{e_{k}}^{m_{k}}\right]=\prod_{i} \mathrm{E}\left[b_{e_{i}}^{m_{i}}\right] & \leq \prod_{\substack{e_{i} \\
e_{i}=\left\{v_{i 1}, v_{i 2}\right\}}} \frac{1}{p^{m_{i}-1}\left(d_{i 1} d_{i 2}\right)^{m_{i} / 2}} \\
& \leq \frac{1}{\left(\prod_{i=1}^{l} d_{a_{i}}\right)\left(p d_{\text {min }}\right)^{2 k-l}},
\end{aligned}
$$

where the $d_{a_{i}}$ term comes from the fact that there must exist an edge contributing $1 /\left(p d_{a_{i}}\right)$ to the product, since an edge incident to $a_{i}$ must occur with multiplicity at least 2 , while all other terms are replaced with their minimum possible values.

Given a set of vertices $S=\left\{v_{1}, \ldots, v_{l+1}\right\}$ and an exposure sequence $\mathbf{e}=$ $\left(a_{1}, \ldots, a_{l}\right)$, let $W(S, \mathbf{e}, k)$ denote the number of surviving walks of length $2 k$ on vertices $S$ with exposure sequence e. We can upper bound the number of surviving walks by the number of surviving walks on these vertices in a complete graph of the same size. Let $W^{\prime}(k, l)$ denote the number of surviving walks of length $2 k$ on the complete graph $K_{l+1}$ such that the vertices are visited in the order $v_{1}, \ldots, v_{l+1}$. (Clearly the labeling does not affect the number of paths, just the fact that the vertices are visited in a particular order.) We note that for a given set $S$ with $|S|=l+1$, there can be at most $l$ ! exposure sequences. Furthermore, for a set $S$ with $l+1$ vertices and an exposure sequence e, we have

$$
W(S, \mathbf{e}, k) \leq W^{\prime}(k, l) .
$$

This inequality is immediate, as each surviving walk on $\left.G\right|_{S}$ corresponds injectively to a walk on the complete graph $K_{l+1}$. Further, note that this inequality holds independently of the exposure sequence e.

Füredi and Komlós [Füredi and Komlós 81] gave an upper bound on $W^{\prime}(k, l)$. Recently, this bound was improved by $\mathrm{Vu}$, and it is this new bound that we use. Inequality (9) in $\mathrm{Vu}[\mathrm{Vu} 07]$ asserts

Lemma 3.2.

$$
W^{\prime}(k, l) \leq\left(\begin{array}{c}
2 k \\
2 l
\end{array}\right) 2^{k+2(k-l)+1}(l+1)^{3(k-l)} .
$$

We can now bound the expected value of $\operatorname{Trace}\left(B^{2 k}\right)$ by both applying the above bound for $W^{\prime}(k, l)$ and using the fact that we are counting surviving walks. We use the notation $u \sim v$ to denote that $u$ and $v$ are adjacent in $G$ :

$$
\begin{aligned}
& \mathrm{E}\left[\operatorname{Trace}\left(B^{2 k}\right)\right] \\
& \leq \sum_{l=1}^{k} \sum_{S=\left\{v_{1}, \ldots, v_{l+1}\right\}} \sum_{\mathbf{e}=\left(a_{1}, \ldots, a_{l}\right)} W(S, \mathbf{e}, k) \frac{1}{\left(\prod_{i=1}^{l} d_{a_{i}}\right)\left(p d_{\min }\right)^{2 k-l}}
\end{aligned}
$$




$$
\begin{aligned}
& \leq \sum_{l=1}^{k} \sum_{\mathbf{e}=\left(a_{1}, \ldots, a_{l}\right)} \sum_{v_{1}} \sum_{v_{2} \sim v_{a_{1}}} \sum_{v_{3} \sim v_{a_{2}}} \ldots \sum_{v_{l+1} \sim v_{a_{l}}} W(S, \mathbf{e}, k) \frac{1}{\left(\prod_{i=1}^{l} d_{a_{i}}\right)\left(p d_{\text {min }}\right)^{2 k-l}} \\
& \leq \sum_{l=1}^{k} \sum_{\mathbf{e}=\left(a_{1}, \ldots, a_{l}\right)} \sum_{v_{1}} \sum_{v_{2} \sim v_{a_{1}}} \sum_{v_{3} \sim v_{a_{2}}} \cdots \sum_{v_{l+1} \sim v_{a_{l}}} W^{\prime}(k, l) \frac{1}{\left(\prod_{i=1}^{l} d_{a_{i}}\right)\left(p d_{\min }\right)^{2 k-l}} \\
& =\sum_{l=1}^{k} \sum_{\mathbf{e}=\left(a_{1}, \ldots, a_{l}\right)} \sum_{v_{1}} \sum_{v_{2} \sim v_{a_{1}}} \sum_{v_{3} \sim v_{a_{2}}} \cdots \sum_{v_{l} \sim v_{a_{l}-1}} W^{\prime}(k, l) \frac{1}{\left(\prod_{i=1}^{l-1} d_{a_{i}}\right)\left(p d_{\min }\right)^{2 k-l}} \\
& =\sum_{l=1}^{k} \sum_{\mathbf{e}=\left(a_{1}, \ldots, a_{l}\right)} \sum_{v_{1}} W^{\prime}(k, l) \frac{1}{\left(p d_{\min }\right)^{2 k-l}} \\
& \leq \sum_{l=1}^{k} \sum_{\mathbf{e}=\left(a_{1}, \ldots, a_{l}\right)} n\left(\begin{array}{c}
2 k \\
2 l
\end{array}\right) 2^{k+2(k-l)+1}(l+1)^{3(k-l)} \frac{1}{\left(p d_{\text {min }}\right)^{2 k-l}} \\
& \leq \sum_{l=1}^{k} l ! n\left(\begin{array}{c}
2 k \\
2 l
\end{array}\right) 2^{3 k}(l+1)^{3(k-l)} \frac{1}{\left(p d_{\min }\right)^{2 k-l}} \\
& \leq \sum_{l=1}^{k} l^{l} n\left(\begin{array}{c}
2 k \\
2 l
\end{array}\right) 2^{3 k}(l+1)^{3(k-l)} \frac{1}{\left(p d_{\min }\right)^{2 k-l}} \\
& \leq \sum_{l=1}^{k} n 32^{k}(l+1)^{3 k-2 l} \frac{1}{\left(p d_{\min }\right)^{2 k-l}} \\
& =\sum_{l=1}^{k} n 32^{k} s_{l, k},
\end{aligned}
$$

where we define

$$
s_{l, k}=\frac{(l+1)^{3 k-2 l}}{\left(p d_{\min }\right)^{2 k-l}} .
$$

For a fixed $\epsilon>0$, with $\epsilon<1 / 4$, we now choose

$$
k=\lfloor\log n+\log (1 / \epsilon)\rfloor
$$

and set

$$
p d_{\min }=\alpha\left(\frac{k}{\log k}\right)^{3 / 2} .
$$

Note that $\alpha$ is a function of $k$ (and hence $n$ ). We wish to show the following: 
Claim

$$
s_{l, k} \leq\left(\frac{c}{\alpha \min \left\{\alpha, \frac{k^{1 / 2}}{\log ^{3 / 2} k}\right\}}\right)^{k}
$$

for some absolute constant $c$.

We let

$$
\begin{aligned}
f(l):=\frac{s_{l, k}}{s_{l-1, k}} & =\frac{\left(1+l^{-1}\right)^{3 k-2 l} p d_{\min }}{l^{2}} \\
& =c_{0} \frac{e^{3 k / l} p d_{\min }}{l^{2}}
\end{aligned}
$$

where $c_{0}$ is upper- and lower-bounded by some absolute constants. For a given value of $p d_{\min }$ and for the range of $0 \leq l \leq k$, the function $s_{l, k}$ either attains its maximum at $l_{0}$ satisfying $l_{0}=k$ and $f(k)>1$, or $l_{0}$ is one of the two integers closest to the solution of $f(l)=1$. Note that, for the first case, we have

$$
\begin{aligned}
s_{l_{0}, k}=s_{k, k} & \leq c\left(\frac{k+1}{p d_{\min }}\right)^{k} \\
& \leq c\left(\frac{k}{\alpha k^{3 / 2} \log ^{-3 / 2} k}\right)^{k} \\
& =c\left(\frac{1}{\alpha k^{1 / 2} \log ^{-3 / 2} k}\right)^{k},
\end{aligned}
$$

which implies (3.2). We may assume that $l_{0}$ is one of the two integers closest to the solution of $f(l)=1$. Furthermore, for $l<k /(2 \log k)$, we have, from (3.3),

$$
\frac{s_{l, k}}{s_{l-1, k}}>\frac{e^{2 \log k}}{k^{2}}>1 .
$$

Therefore, we may assume that

$$
l_{0} \geq \frac{k}{2 \log k} .
$$

There are two possibilities:

- Case I. $l_{0} \leq 100 k / \log k$. Then

$$
\begin{aligned}
s_{l_{0}, k} & \leq\left(c^{\prime} \frac{l_{0}^{3}}{\left(p d_{\min }\right)^{2}}\right)^{k} \\
& \leq\left(c \frac{k^{3} /(\log k)^{3}}{\alpha^{2}(k / \log k)^{3}}\right)^{k} \\
& =\left(\frac{c}{\alpha^{2}}\right)^{k}
\end{aligned}
$$

which implies (3.2). 
- Case 2. $l_{0} \geq 100 k / \log k$. We use the fact that $l_{0}$ is one of the two integers closest to the solution of $f(l)=1$. From equation (3.3), we have

$$
\begin{aligned}
s_{l_{0}, k} & \leq\left(\frac{l_{0}^{3-2 l_{0} / k}}{\left(p d_{\min }\right)^{2-l_{0} / k}}\right)^{k} \\
& \leq\left(c^{\prime \prime} \frac{l_{0}^{3-2 l_{0} / k}}{\left(l_{0}^{2} e^{-3 k / l_{0}}\right)^{2-l_{0} / k}}\right)^{k} \\
& \leq\left(\frac{c^{\prime \prime \prime} e^{6 k / l_{0}}}{l_{0}}\right)^{k} .
\end{aligned}
$$

One can check that, for the given range of $l_{0}$, this satisfies

$$
\begin{aligned}
S_{l_{0}, k} & \leq\left(\frac{c^{\prime \prime \prime} k}{l_{0}^{2}}\right)^{k} \\
& \leq\left(\frac{c^{\prime \prime \prime} k e^{3 k / l_{0}}}{l_{0}^{2}}\right)^{k} \\
& \leq\left(\frac{c_{0}}{\alpha k^{1 / 2} \log ^{-3 / 2} k}\right)^{k},
\end{aligned}
$$

which again implies (3.2).

In this section, $c_{0}, c, c^{\prime}, c^{\prime \prime}, \ldots$ are suitably chosen integers. The proof for the claim is completed.

We are now ready to consider bounding the norm of $B$.

$$
\begin{aligned}
\mathrm{E}\left[\operatorname{Trace}\left(B^{2 k}\right)\right] & \leq n^{2} 32^{k} \max _{l} s_{l, k} \\
& \leq 2 n^{2}\left(\frac{32 c_{0}}{\alpha \min \left\{\alpha, k^{1 / 2} \log ^{-3 / 2} k\right\}}\right)^{k} .
\end{aligned}
$$

Since $\mathrm{E}\left[\|B\|^{2 k}\right] \leq \mathrm{E}\left[\operatorname{Trace}\left(B^{2 k}\right)\right]$, we have

$$
\mathrm{E}\left[\|B\|^{2 k}\right] \leq 2 n^{2}\left(\frac{c}{\min \left\{\alpha, \alpha^{1 / 2} k^{1 / 4} \log ^{-3 / 4} k\right\}}\right)^{2 k} .
$$

By the previous equation and Markov's equality, we have

$$
\begin{aligned}
& \operatorname{Pr}\left(\|B\| \geq 2 \frac{c}{\min \left\{\alpha, \alpha^{1 / 2} k^{1 / 4} \log ^{-3 / 4} k\right\}}\right) \\
& \quad=\operatorname{Pr}\left(\|B\|^{2 k} \geq 2^{2 k}\left(\frac{c}{\min \left\{\alpha, \alpha^{1 / 2} k^{1 / 4} \log ^{-3 / 4} k\right\}}\right)^{2 k}\right)
\end{aligned}
$$




$$
\begin{aligned}
& \leq \frac{\mathrm{E}\left[\|B\|^{2 k}\right]}{2^{2 k}\left(\frac{c}{\min \left\{\alpha, \alpha^{1 / 2} k^{1 / 4} \log ^{-3 / 4} k\right\}}\right)^{2 k}} \\
& \leq \frac{2 n^{2}\left(\frac{c}{\min \left\{\alpha, \alpha^{1 / 2} k^{1 / 4} \log ^{-3 / 4} k\right\}}\right)^{2 k}}{2^{2 k}\left(\frac{c}{\min \left\{\alpha, \alpha^{1 / 2} k^{1 / 4} \log ^{-3 / 4} k\right\}}\right)^{2 k}} \\
& \leq \frac{2 n^{2}}{2^{2 k}} \\
& \leq \epsilon
\end{aligned}
$$

for the given $\epsilon>0$ (noting that this holds as $\epsilon<1 / 4$ ) and our choice of $k$ in (3.1). Hence we have proved that almost surely we have

$$
\|C\| \leq\left\|M^{\prime}\right\|+\|B\|=1-\lambda+O\left(\frac{1}{\min \left\{\alpha, \alpha^{1 / 2} k^{1 / 4} \log ^{-3 / 4} k\right\}}\right) .
$$

In a similar way, we can use the fact that $\|C\| \geq\left\|M^{\prime}\right\|-\|B\|$ to get

$$
\|C\|=1-\lambda \pm O\left(\frac{1}{\min \left\{\alpha, \alpha^{1 / 2} k^{1 / 4} \log ^{-3 / 4} k\right\}}\right) .
$$

It is easily verified that

$$
O\left(\frac{1}{\min \left\{\alpha, \alpha^{1 / 2} k^{1 / 4} \log ^{-3 / 4} k\right\}}\right)=O\left(\sqrt{\frac{\log n}{p d_{\min }}}+\frac{(\log n)^{3 / 2}}{p d_{\min }(\log \log n)^{3 / 2}}\right)
$$

which completes the proof of Theorem 2 .

\section{Bounding the Spectral Gap}

In this section, we plan to give a complete proof for Theorem 1. Namely, we wish to show that, for a graph $G$ with spectral gap $\lambda$ and minimum degree $d_{\text {min }}$, a random subgraph $H$ obtained from $G$ with edge-selection probability $p$ almost surely has eigenvalues of the Laplacian $\mathcal{L}_{H}$ of $H$ satisfying

$$
\lambda_{H}=\max _{i \neq 0}\left|1-\eta_{i}\right|=1-\lambda+O\left(\sqrt{\frac{\log n}{p d_{\min }}}+\frac{(\log n)^{3 / 2}}{p d_{\min }(\log \log n)^{3 / 2}}\right) .
$$

As a matter of notation, we let $d_{i}$ refer to the degree of vertex $v_{i}$ in $G$ and $d_{i}^{\prime}$ refer to the degree of vertex $i$ in $H$. We also let $a_{i j}^{\prime}$ refer to the $i j$ th entry of $A_{H}$, the adjacency matrix of $H$. 
To prove Theorem 1, recall that the eigenvalues of the Laplacian $\mathcal{L}_{H}$ satisfy

$$
\max _{i \neq 0}\left|1-\eta_{i}\right|=|| M \|
$$

where $M=D_{H}^{-1 / 2} A_{H} D_{H}^{-1 / 2}-\frac{1}{\operatorname{vol}(H)} D_{H}^{1 / 2} K D_{H}^{1 / 2}$. Now we write

$$
M=E+C+R+S,
$$

where we define

$$
\begin{aligned}
E= & D_{H}^{-1 / 2} A_{H} D_{H}^{-1 / 2}-\frac{1}{p} D^{-1 / 2} A_{H} D^{-1 / 2}-\frac{p}{\operatorname{vol}(G)} D D_{H}^{-1 / 2} K D_{H}^{-1 / 2} D \\
& +\frac{1}{\operatorname{vol}(G)} D^{1 / 2} K D^{1 / 2} \\
R= & \frac{p}{\operatorname{vol}(G)} D D_{H}^{-1 / 2} K D_{H}^{-1 / 2} D-\frac{1}{p \operatorname{vol}(G)} D_{H}^{1 / 2} K D_{H}^{1 / 2} \\
S= & \left(\frac{1}{p \operatorname{vol}(G)}-\frac{1}{\operatorname{vol}(H)}\right) D_{H}^{1 / 2} K D_{H}^{1 / 2}
\end{aligned}
$$

and $C$ is as defined in (2.1). Thus

$$
\begin{aligned}
e_{i j} & =\left(a_{i j}^{\prime}-\frac{p d_{i} d_{j}}{\operatorname{vol}(G)}\right)\left(\frac{1}{\sqrt{d_{i}^{\prime} d_{j}^{\prime}}}-\frac{1}{p \sqrt{d_{i} d_{j}}}\right) \\
r_{i j} & =\frac{1}{p \operatorname{vol}(G)} \frac{p^{2} d_{i} d_{j}-d_{i}^{\prime} d_{j}^{\prime}}{\sqrt{d_{i}^{\prime} d_{j}^{\prime}}} \\
s_{i j} & =\left(\frac{1}{p \operatorname{vol}(G)}-\frac{1}{\operatorname{vol}(H)}\right) \sqrt{d_{i}^{\prime} d_{j}^{\prime}}
\end{aligned}
$$

and

$$
c_{i j}=\frac{a_{i j}^{\prime}}{p \sqrt{d_{i} d_{j}}}-\frac{1}{\operatorname{vol}(G)} \sqrt{d_{i} d_{j}} .
$$

Clearly,

$$
\|M\| \leq\|E\|+\|C\|+\|R\|+\|S\|
$$

Hence, it suffices to establish the appropriate upper bounds for the norms of $E, C, R$, and $S$.

To bound these, we use the following Chernoff bounds (see, e.g., [Chung and $\mathrm{Lu} 06])$. 
Lemma 4.I. For $1 \leq i \leq n$, let $X_{i}$ be independent random variables satisfying $\left|X_{i}\right| \leq M$. Let $X=\sum_{i} X_{i}$. Then we have, for any $a>0$,

$$
P(|X-E[X]|>a) \leq e^{-\frac{a^{2}}{2(\operatorname{Var}(X)+M a / 3)}} .
$$

We will prove the following lemma (whose proof we delay until after the proof of Theorem 1).

Lemma 4.2. Assuming that $p d_{\min } \gg \log n$, almost surely every vertex $v_{i}$ satisfies

$$
d_{i}^{\prime}=p d_{i}\left(1+O\left(\sqrt{\frac{\log n}{p d_{\min }}}\right)\right) .
$$

Let $X_{e}$, for $e \in E(G)$, be the random indicator variable that is 1 if $e \in H$ and 0 otherwise. We can write

$$
\operatorname{vol}(H)=\sum_{e \in E(G)} 2 X_{e}
$$

We can show that almost surely

$$
|\operatorname{vol}(H)-p \operatorname{vol}(G)|<2 \sqrt{p \operatorname{vol}(H)} g(n)
$$

for any function $g(n)$ that goes to infinity as $n$ approaches infinity.

We also have the following lemma (whose proof will be given later).

Lemma 4.3. Suppose that $p d_{\min } \gg \log n$. Almost surely the vector $\chi$ with $\chi(i)=$ $\left(d_{i}^{\prime}-p d_{i}\right) / \sqrt{p d_{i}}$ satisfies

$$
\|\chi\|^{2} \leq(1+o(1)) n
$$

Proof of Theorem I. We note that we established a bound on $\|C\|$ in the last section. By Theorem 2, we have that almost surely

$$
\|C\|=1-\lambda+O\left(\sqrt{\frac{\log n}{p d_{\min }}}+\frac{(\log n)^{3 / 2}}{p d_{\min }(\log \log n)^{3 / 2}}\right) .
$$

For convenience, we define

$$
\beta=\sqrt{\frac{\log n}{p d_{\min }}}+\frac{(\log n)^{3 / 2}}{p d_{\min }(\log \log n)^{3 / 2}} .
$$


For $\|R\|$, almost surely we have the following by using equation (4.1) and the Cauchy-Schwartz inequality:

$$
\begin{aligned}
\|R\|= & \max _{\|y\|=1}\langle y, R y\rangle \\
\leq & \max _{\|y\|=1} \frac{1}{p \operatorname{vol}(G)} \sum_{i, j} y_{i} y_{j} \frac{d_{i}^{\prime}\left(d_{j}^{\prime}-p d_{j}\right)+\left(d_{i}^{\prime}-p d_{i}\right) p d_{j}}{\sqrt{d_{i}^{\prime} d_{j}^{\prime}}} \\
= & \frac{1}{p \operatorname{vol}(G)} \max _{\|y\|=1}\left\{\sum_{i} \sqrt{d_{i}^{\prime} y_{i}} \sum_{j} \frac{\left(d_{j}^{\prime}-p d_{j}\right) y_{j}}{\sqrt{d_{j}^{\prime}}}\right. \\
& +\sum_{i} \frac{\left(d_{i}^{\prime}-p d_{i}\right) y_{i}}{\left.\sqrt{d_{i}^{\prime}} \sum_{j} \frac{p d_{j} y_{j}}{\sqrt{d_{j}^{\prime}}}\right\}} \\
\leq & \frac{1}{p \operatorname{vol}(G)} \max _{\|y\|=1}\left\{\left(\sum_{i} d_{i}^{\prime}\right)^{1 / 2}\|y\|\left(\sum_{j} \frac{\left(d_{j}^{\prime}-p d_{j}\right)^{2}}{d_{j}^{\prime}}\right)^{1 / 2}\right. \\
& \left.+\|y\|\left(\sum_{j} \frac{\left(d_{j}^{\prime}-p d_{j}\right)^{2}}{d_{j}^{\prime}}\right)^{1 / 2}\left(\sum_{j} \frac{\left(p d_{j}\right)^{2}}{d_{j}^{\prime}}\right)^{1 / 2}\right\} \\
\leq & (2+o(1)) \sqrt{\frac{n}{p \operatorname{vol}(G)}} \\
\leq & (1+o(1)) \frac{2}{\sqrt{p d}} \\
= & o(\beta) .
\end{aligned}
$$

For $\|S\|$, by using (4.2) and the Cauchy-Schwartz inequality, we have

$$
\begin{aligned}
\|S\| & =\max _{\|y\|=1}\langle y, S y\rangle=\max _{\|y\|=1} \sum_{i, j}\left|y_{i} y_{j}\left(\frac{1}{p \operatorname{vol}(G)}-\frac{1}{\operatorname{vol}(H)}\right)\right| \sqrt{d_{i}^{\prime} d_{j}^{\prime}} \\
& \leq\left(\frac{1}{p \operatorname{vol}(G)}-\frac{1}{\operatorname{vol}(H)}\right) \max _{\|y\|=1} \sum_{i, j}\left|y_{i} \sqrt{d_{i}^{\prime}}\right|\left|y_{j} \sqrt{d_{j}^{\prime}}\right| \\
& \leq \frac{2 \sqrt{p \operatorname{vol}(G) \log (n)}}{p \operatorname{vol}(H) \operatorname{vol}(G)} \max _{\|y\|=1}\left(\sum_{i} \mid y_{i} \sqrt{d_{i}^{\prime} \mid}\right)^{2} \\
& \leq \frac{2 \sqrt{p \operatorname{vol}(G) \log (n)}}{p \operatorname{vol}(H) \operatorname{vol}(G)}\left(\sum_{i} y_{i}^{2}\right)\left(\sum_{i} d_{i}^{\prime}\right) \\
& =O\left(\sqrt{\frac{\log n}{p \operatorname{vol}(G)}}\|y\|^{2}\right)
\end{aligned}
$$




$$
\begin{aligned}
& =o\left(\frac{\log n}{\sqrt{p d_{\text {min }}}}\right) \\
& =o(\beta) .
\end{aligned}
$$

Finally, it remains to bound $\|E\|$. We recall

$$
\begin{aligned}
e_{i j} & =\left(a_{i j}^{\prime}-\frac{p d_{i} d_{j}}{\operatorname{vol}(G)}\right)\left(\frac{1}{\sqrt{d_{i}^{\prime} d_{j}^{\prime}}}-\frac{1}{p \sqrt{d_{i} d_{j}}}\right) \\
& =c_{i j} \frac{p \sqrt{d_{i} d_{j}}-\sqrt{d_{i}^{\prime} d_{j}^{\prime}}}{\sqrt{d_{i}^{\prime} d_{j}^{\prime}}} .
\end{aligned}
$$

Thus, we have

$$
\begin{aligned}
\|E\| & =\max _{\|y\|=1}\langle y, E y\rangle \\
& =\max _{\|y\|=1} \sum_{i, j} y_{i} y_{j} c_{i, j} \frac{\sqrt{d_{i}^{\prime}}\left(\sqrt{d_{j}^{\prime}}-\sqrt{p d_{j}}\right)+\left(\sqrt{d_{i}^{\prime}}-\sqrt{p d_{i}}\right) \sqrt{p d_{j}}}{\sqrt{d_{i}^{\prime} d_{j}^{\prime}}} .
\end{aligned}
$$

Let $y_{i}^{\prime}=y_{i}\left(\sqrt{d_{i}^{\prime}}-\sqrt{p d_{i}}\right) / \sqrt{d_{i}^{\prime}}$ and $y_{i}^{\prime \prime}=y_{i} \sqrt{p d_{i}} / \sqrt{d_{i}^{\prime}}$. Then we have almost surely

$$
\begin{aligned}
\|E\| & \leq \max _{\|y\|=1}\left\langle y, C y^{\prime}\right\rangle+\left\langle y^{\prime}, C y^{\prime \prime}\right\rangle \\
& \leq \max _{\|y\|=1}\|C\|\left\|y^{\prime}\right\|+\|C\|\left\|y^{\prime}\right\|\left\|y^{\prime \prime}\right\| \\
& \leq O(\beta) .
\end{aligned}
$$

This last observation follows from Lemma 3, which implies

$$
\left\|y^{\prime}\right\| \leq\|y\|\left(1-\frac{1}{\sqrt{1+O\left(\sqrt{\log n / p d_{\min }}\right)}}\right)=O(\beta)
$$

and $\left\|y^{\prime \prime}\right\|=(1+o(1))\|y\|=O(1)$. Note that we have already observed that $\|C\|=O(1)$. Combining these results, we have

$$
\begin{aligned}
\max _{i \neq 0}\left|1-\eta_{i}\right| & =\|M\| \\
& \leq\|E\|+\|C\|+\|R\|+\|S\| \\
& \leq 1-\lambda+O(\beta) .
\end{aligned}
$$

In the other direction, the lower bound follows as $\|M\| \geq\|C\|-\|E\|-\|R\|-$ $\|S\|=1-\lambda+O(\beta)$. 
This gives the following bound on the spectral gap of $H$, completing the proof of Theorem 1:

$$
\lambda_{H}=1-\max _{i \neq 0}\left|1-\eta_{i}\right|=\lambda-O(\beta) .
$$

It remains to prove Lemmas 3 and 4 .

Proof of Lemma 3. For a vertex $v_{i} \in G$, we can write $d_{i}^{\prime}=\sum_{v_{j} \sim v_{i}} X_{j}$ where $X_{i}$ is the random indicator variable having value 1 if $\left\{v_{i}, v_{j}\right\} \in E(H)$ and 0 otherwise. Then $\mathrm{E}\left[d_{i}^{\prime}\right]=p d_{i}$ and $\operatorname{Var}\left(d_{i}^{\prime}\right)=d_{i} p(1-p)$. By the Chernoff bounds, we have

$$
\operatorname{Pr}\left(\left|d_{i}^{\prime}-p d_{i}\right|>a\right) \leq \exp \left(-\frac{a^{2}}{2\left(d_{i} p(1-p)\right)+a / 3}\right)
$$

Setting $a=2 \sqrt{\log (n) p d_{i}}$, we have that

$$
\begin{aligned}
\operatorname{Pr}\left(\left|d_{i}^{\prime}-p d_{i}\right|>a\right) & \leq \exp \left(-\frac{4 p d_{i} \log (n)}{2 p d_{i}(1-p)+4 \sqrt{p d_{i} \log (n)} / 3}\right) \\
& \leq n^{-2+o(1)} .
\end{aligned}
$$

Thus, almost surely, for all $i$ we have $\left|d_{i}^{\prime}-p d_{i}\right| \leq 2 \sqrt{\log (n) p d_{i}}$. This can be restated as, for all $i$,

$$
\left|d_{i}^{\prime}-p d_{i}\right| \leq p d_{i} \sqrt{\frac{\log n}{p d_{i}}}=p d_{i} O\left(\sqrt{\frac{\log n}{p d_{\min }}}\right) .
$$

The following proof of Lemma 4 is analogous to Lemma 3.3 in [Chung et al. 04].

Proof of Lemma 4. For a vertex $v_{i} \in G$, let $X_{i}=\left(d_{i}^{\prime}-p d_{i}\right)^{2}$ and $X=\sum_{i=1}^{n} \frac{X_{i}}{p d_{i}}$. For each $i$, we can write

$$
X_{i}=\left(\sum_{v_{j} \sim v_{i}} X_{i j}-p\right)^{2}
$$

where the $X_{i j}$ 's are the indicator random variables of the event that $v_{i}$ is adjacent to $v_{j}$ in $H$ (as denoted by $v_{i} \sim v_{j}$ ). We define

$$
x_{i j}= \begin{cases}X_{i j}-p & \text { if } v_{i} \sim v_{j} \\ 0 & \text { otherwise }\end{cases}
$$


Thus, $E\left[x_{i j}\right]=0$, and $X_{i}=\left(\sum_{j} x_{i j}\right)^{2}$. Also,

$$
\begin{aligned}
\mathrm{E}\left[X_{i}\right] & =\operatorname{Var}\left(d_{i}^{\prime}\right)=\mathrm{E}\left[\sum_{v_{i} \sim v_{j}} x_{i j}^{2}\right]<p d_{i} \\
\mathrm{E}\left[X_{i}^{2}\right] & =\mathrm{E}\left[\left(d_{i}^{\prime}-p d_{i}\right)^{4}\right] \\
& =\mathrm{E}\left[\left(\sum_{v_{i} \sim v_{j}} x_{i j}\right)^{4}\right] \\
& =\sum_{i \sim j} \mathrm{E}\left[x_{i j}^{4}\right]+6 \sum_{\substack{j \neq k \\
v_{j} \sim v_{i}, v_{k} \sim v_{i}}} \mathrm{E}\left[x_{i j}^{2} x_{i k}^{2}\right] \\
& \leq p d_{i}+6 p^{2} d_{i}^{2} .
\end{aligned}
$$

If $v_{i} \not v_{j}$ and $v_{i} \neq v_{j}$, then $X_{i}$ and $X_{j}$ are independent. If $v_{i} \sim v_{j}$ and $v_{i} \neq v_{j}$, we have

$$
\begin{aligned}
\mathrm{E}\left[X_{i} X_{j}\right] & =\mathrm{E}\left[\left(d_{i}^{\prime}-p d_{i}\right)^{2}\left(d_{j}^{\prime}-p d_{j}\right)^{2}\right] \\
& =\mathrm{E}\left[\left(\sum_{v_{k} \sim v_{i}} x_{i k}\right)^{2}\left(\sum_{v_{l} \sim v_{j}} x_{l j}\right)^{2}\right] \\
& =\sum_{\substack{v_{k} \sim v_{i}, v_{l} \sim v_{j} \\
\{i, k\} \neq\{l, j\}}} \mathrm{E}\left[v_{i k}^{2} v_{l j}^{2}\right]+\mathrm{E}\left[v_{i j}^{4}\right] \\
& \leq p^{2} d_{i} d_{j}+p .
\end{aligned}
$$

Thus,

$$
\begin{aligned}
\operatorname{Var}\left(X_{i}\right) & \leq p d_{i}+5 p^{2} d_{i}^{2} \\
\operatorname{coVar}\left(X_{i}, X_{j}\right) & \leq \begin{cases}0 & \text { if } v_{i} \nsim v_{j} \\
p & \text { otherwise. }\end{cases}
\end{aligned}
$$

Therefore

$$
\begin{aligned}
\mathrm{E}[X] & =\sum_{i=1}^{n} \frac{1}{p d_{i}} \mathrm{E}\left[X_{i}\right]<n \\
\operatorname{Var}(X) & =\sum_{i=1}^{n} \frac{1}{p^{2} d_{i}^{2}} \operatorname{Var}\left(X_{i}\right)+2 \sum_{i<j \leq n} \frac{1}{w_{i} w_{j}} \operatorname{coVar}\left(X_{i}, X_{j}\right) \\
& \leq\left(5+\frac{1}{p d_{\text {min }}}+\frac{1}{d}\right) n \\
& \leq 6 n .
\end{aligned}
$$

Using Chebyshev's inequality, we have, for any $a>0$,

$$
\operatorname{Pr}(|X-E[X]|>a) \leq \frac{\operatorname{Var}(X)}{a^{2}}
$$


Setting $a=\sqrt{n} g(n)$, with $g(n) \gg 1$, then almost surely we have $X \leq(1+o(1)) n$. From the definition, $\|\chi\|^{2}=X$. Thus, almost surely

$$
\|\chi\|^{2} \leq(1+o(1)) n
$$

as desired.

\section{Concluding Remarks}

In this paper, we examine the spectral relationship between a host graph $G$ and its random subgraph with edge-selection probability $p$. If $G$ has $n$ vertices with a spectral gap $\lambda$ and minimum degree $d_{\text {min }}$, then we prove that a random subgraph of $G$ on $n$ vertices with edge-selection probability $p$ almost surely has a spectral gap of $\lambda-O\left(\sqrt{\frac{\log n}{p d_{\min }}}+\frac{(\log n)^{3 / 2}}{p d_{\min }(\log \log n)^{3 / 2}}\right)$. The special case of having the host graph as the complete graph on $n$ vertices and a random subgraph $H$ chosen with edge-selection probability $p$ is the Erdös-Rényi graph $G(n, p)$. Since the complete graph $K_{n}$ has eigenvalue $\lambda_{1}=n /(n-1)$, our bound for $\lambda_{H}$ is $\left|1-\lambda_{H}\right|=O(\sqrt{(\log n) / n})$, which is off by a factor of $\sqrt{\log n}$ of the best-known spectral bound for $G(n, p)$. Therefore, there is room for improvements (e.g., by a factor of $\sqrt{\log n}$ ) concerning the statements of the main theorem here.

Acknowledgments. The research of the first author was supported in part by NSF Grants DMS 0457215 and ITR 0426858.

\section{References}

[Alon et al. 04] N. Alon, I. Benjamini, and A. Stacey. "Percolation on Finite Graphs and Isoperimetric Inequalities." Annals of Probability 32:3 (2004), 1727-1745.

[Chung 97] F. Chung. Spectral Graph Theory. Providence, RI: AMS Publications, 1997.

[Chung et al. 04] F. Chung, L. Lu, and V. Vu. "The Spectra of Random Graphs with Given Expected Degrees." Internet Mathematics 1:3 (2004), 257-275.

[Chung and Lu 06] F. Chung and L. Lu. Complex Graphs and Networks. Providence, RI: AMS Publications, 2006.

[Friedman 03] J. Friedman. "A Proof of Alon's Second Eigenvalue Conjecture." In Proceedings of the Thirty-Fifth Annual ACM Symposium on Theory of Computing, pp. 720-724. New York: ACM Press, 2003.

[Füredi and Komlós 81] Z. Füredi and J. Komlós. "The Eigenvalues of Random Symmetric Matrices." Combinatorica 1:3 (1981), 233-241.

[Frize et al. 04] A. Frize, M. Krivelevich, and R. Martin. "The Emergence of a Giant Component of Pseudo-Random Graphs." Random Structures and Algorithms 24:1 (2004), 42-50. 
[Krivelevich and Sudakov 06] M. Krivelevich and B. Sudakov. "Pseudo-Random Graphs." In More Sets, Graphs and Numbers, pp. 199-262. Bolyai Society Mathematical Studies 15. Berlin-Heidlberg: Springer-Verlag, 2006.

[Nachmias and Peres 08] A. Nachmias and Y. Peres. "Critical Random Graphs: Diameter and Mixing Time." Annals of Probability 36:4 (2008), 1267-1286.

[Ofek 07] E. Ofek. "On the Expansion of the Giant Component in Percolated $(n, d, \lambda)$ Graphs." Combinatorics, Probability and Computing 16:3 (2007), 445-457.

[Vu 07] V. Vu. "Spectral Norm of Random Matrices." Combinatorica 27:6 (2007), 721736.

[Wigner 58] E. P. Wigner. "On the Distribution of the Roots of Certain Symmetric Matrices." The Annals of Mathematics 67 (1958), 325-327.

Fan Chung, Department of Mathematics, University of California, San Diego, 9500 Gilman Drive, 0112, La Jolla, CA 92093-0112 (fan@ucsd.edu)

Paul Horn, Department of Mathematics, University of California, San Diego, 9500 Gilman Drive, 0112, La Jolla, CA 92093-0112 (phorn@math.ucsd.edu)

Received May 11, 2007; accepted October 22, 2007. 